\title{
Role of 12-lipoxygenase derived eicosanoids on epithelial barrier function in intestinal Caco-2 cells
}

\author{
M. J. Rodríguez-lagunas, J. J. Moreno and R. Ferrer \\ Departament de Fisiologia, Facultat de Farmàcia, Universitat de Barcelona, Av. Joan XXIII s/n Spain
}

Eicosanoids are lipid mediators synthesized by the arachidonic acid (AA) cascade from membrane polyunsaturated fatty acids (PUFA) trough three distinct pathways: cyclooxygenase, lipooxygenase (LOX) and cytochrome P450 pathways. Eicosapentaenoic acid, an $n$-3 PUFA, is also substrate of the enzymes of the AA cascade. Thus, 5-LOX gives rise to the 5-series leukotrienes, and 12-LOX generates 12-HEPE, the homologue of 12-HETE when the source is AA. Fish oil supplementation has been used as a preventive measure against a number of diseases including coronary heart disease, cancer, and inflammatory bowel disease (IBD). IBD and other intestinal diseases are associated with the disruption of epithelial barrier function ${ }^{(1)}$. In this sense, we recently demonstrated that $\mathrm{PGE}_{2}$, which is increased in the mucosa of IBD patients, induces the disruption of this barrier in differentiated Caco- 2 cells ${ }^{(2)}$. In this line, the present study aims to investigate the potential of 12-LOX derived eicosanoids 12-HETE and 12-HEPE to disrupt paracellular permeability (PP) and if so, the mechanism implicated.

PP permeability was assessed from transepithelial electrical resistance (TER) and apical to basolateral dextran fluxes in Caco-2 cells grown on polycarbonate filters for 21 days. The cultures were maintained for $3 \mathrm{~h}$ with the eicosanoids in the apical and basolateral compartments. Intracellular $\mathrm{Ca}^{2+}$ concentration was monitored using Fura 2-AM, and cAMP determination and NFkB activation were performed using competitive EIA kits. Tight junction proteins were studied using fluorescent microscopy.

The results indicate that both 12-HETE enantiomers altered PP by decreasing TER and increasing dextran fluxes whereas the $n-3$ derived eicosanoid 12-HEPE did not modify any of these variables. Furthermore, the results indicate that $\mathrm{Ca}^{2+}$ and $\mathrm{cAMP}$ but not NFKB were the downstream targets of both enantiomers. In addition, tight junction reorganization was observed when studying occludin whereas ZO-1 remained unaltered.

In conclusion, we have demonstrated that 12-(R) and 12-(S)-HETE, eicosanoids derived from $n$-6 PUFA, but not 12-HEPE are able to disrupt epithelial barrier function. This could be, at least in part, an explanation of the beneficial role of fish oil in IBD.

This study was supported by Grant BFU2007-61727/BFI (Ministerio de Ciencia y Tecnología) and 2009SGR438 (Generalitat de Catalunya).

1. Turner JR (2006) Am J Pathol 169, 1901-1909.

2. Rodríguez-Lagunas MJ, Martín-Venegas R, Moreno JJ \& Ferrer R (2010) Am J Physiol Cell Physiol 299, 324-334. 